\title{
Crítica desde el pensamiento complejo a los métodos cuantitativos para el análisis educativo. EI caso de los rendimientos educativos*
}

\author{
Carlos Ricardo Aguilar Astorga ${ }^{1}$ \\ (iD http://orcid.org/0000-0003-3760-8338 \\ Universidad Autónoma Metropolitana, Unidad Lerma, México
}

DOI: http://dx.doi.org/10.17081/eduhum.19.33.2649

Recibido: 23 de junio de 2016

Aceptado: 10 de diciembre de 2016

\section{Criticizing quantitative methods in education from a complex thought view. The case of academic performance}

\author{
Palabras clave: \\ Pensamiento complejo, \\ Rendimientos educativos, \\ Política educativa, \\ Mínimos cuadrados ordinarios.
}

Key words:

Complex thought,

Educational performance,

Educational policy,

Ordinary least squares method.

\begin{abstract}
Resumen
En materia de política educativa es común asociar el nivel salarial de un sujeto respecto de su nivel educativo: sin embargo, dicha relación no es tan lineal como parece, sino que, visto desde el pensamiento complejo, intervienen factores como los costos marginales -a los que cada individuo se enfrenta al asistir a su centro educativo-, los cuales comprueban otra reciprocidad de variables. La forma como se construyen las asignaciones presupuestales en materia educativa en México no se deriva de este hecho, sino que hallan una correlación entre rendimiento educativo y asignación presupuestal por nivel educativo, excluyendo factores explicativos que el pensamiento complejo permite destacar. Tomar esto en consideración, mejoraría la política pública educativa.
\end{abstract}

\begin{abstract}
On the issue of educational policy is commonplace to associate the income level of any subject according to his level of education: However, this relation is not so linear as it seems, but rather, looking at it from a complex thought view, variables like marginal costs- faced by individuals at the moment of attending to his institution, are a proof of the reciprocity of other variables. The way budgets are made for education in Mexico does not follow this fact, but they keep a correlation between educational performance and budget assignment according to the level of education, excluding those explanatory variables that complex thought highlights. Taking this into consideration, it would finally improve educational public policy.
\end{abstract}

\section{(요요 $\Theta$}

Referencia de este artículo (APA): Aguilar, C. (2017). Crítica desde el pensamiento complejo a los métodos cuantitativos para el análisis educativo. El caso de los rendimientos educativos. En Revista Educación y Humanismo, 19(33), 357-368. http://dx.doi.org/ 10.17081/eduhum.19.33.2649

\footnotetext{
* La línea de investigación que subyace el trabajo es: Seminario sobre transdisciplina y complejidad.

1. Profesor-investigador del Departamento de Procesos Sociales, Universidad Autónoma Metropolitana, Unidad Lerma. c.aguilar@correo.ler.uam.mx
} 


\section{Introducción}

Desde el enfoque de la complejidad, el proceso de enseñanza-aprendizaje implica la discusión de saberes, su correlación, interrelación y el reconocimiento de la unidad en lo múltiple, respetando así la diversidad de lo uno en lo variado. El presente trabajo aborda justamente el hecho de que este problema no ha sido tangible o siquiera esperable desde el plano de la política educativa. Por el contrario, la difusión de saberes se ha simplificado a la hiper especialización, a enfoques cada vez más mono-relacionales que no solo los disipan y diluyen sino que, más aún, tecnifican el conocimiento y lo profesionalizan de manera cada vez más aislada. Todo esto tiene vínculos con un proceso político progresivamente segmentado y hegemónico, que excluye a los muchos e incluye cada vez a los menos.

El objetivo de este trabajo consiste, por tanto, en realizar una crítica a los métodos cuantitativos para el análisis educativo utilizando un caso de estudio de rendimientos educativos para la asignación del presupuesto en esta materia, con la finalidad de mostrar los alcances y limitaciones de este tipo de investigaciones en el ámbito de las políticas públicas.

El trabajo dispone de tres apartados. En el primero, se aborda el tema educativo como un fenómeno complejo, alejándolo del reduccionismo lineal que los enfoques cuantitativos le atribuyen. En el segundo, se plantea el caso de estudio, es decir, de los rendimientos educativos de la educación en México para el año 2012, utilizando la herramienta de econometría. Aunado a ello, se discute críticamente el uso de los métodos cuantitativos en el análisis educativo, así como sus implicaciones de política pública. Por último, con la intención de poner en perspectiva el ejercicio realizado, se expresan algunas reflexiones.

\section{La relación educación-complejidad como} problema

En los últimos años, las políticas públicas educativas se han apoyado cada vez más en las herramientas que proporcionan los métodos cuantitativos para la definición, el diseño, la implementación y la evaluación de diversas acciones de gobierno dirigidas a resolver problemas relacionados con la baja calidad educativa, la escasa pertinencia social de los programas y contenidos curriculares, la alta deserción de estudiantes, una cobertura insuficiente, la asignación de presupuestos educativos en un ambiente de escasez, entre otros. En este contexto, vale la pena preguntar: ¿cuáles son los alcances y límites de la observación y análisis de los fenómenos educativos desde la perspectiva estadística?, ¿cambia la reflexión un análisis desde el pensamiento complejo?, ¿qué se puede rescatar del pensamiento de Édgar Morin sobre la unilateralidad reflexiva del tema educativo?

Justamente, Morin(1999), en sus Siete saberes, destaca el concepto de inteligencia ciega: 
(...) destruye los conjuntos y las totalidades, aísla todos sus objetos de sus ambientes. No puede concebir el lazo inseparable entre el observador y la cosa observada. Las realidades clave son desintegradas. Pasan entre los hiatos que separan a las disciplinas. Las disciplinas de las ciencias humanas no necesitan más de la noción de hombre. Y los ciegos pedantes concluyen que la existencia del hombre es solo ilusoria. Mientras los medios producen la cretinización vulgar, la Universidad produce la cretinización de alto nivel. La metodología dominante produce oscurantismo porque no hay más asociación entre los elementos disjuntos del saber y, por lo tanto, tampoco posibilidad de engranarlos y de reflexionar sobre ellos. (Morin, 2008, pp.30-31).

Ahora bien, desde la óptica compleja, la educación implica su relación con otros saberes, pero sobre todo con la sociedad. Para nadie es desconocido que la enseñanza se ha hiperespecializado, imposibilitando un diálogo recíproco entre la parte y el todo. Morin, Ciurana y Motta (2003) proponen siete principios que guían el pensamiento complejo; a continuación se presentan a manera de síntesis:

\section{Sistémico u organizacional. El todo es mayor}

a la suma de las partes, pero a su vez, el todo es menor que las sumas de las partes. Las políticas educativas -en tanto programas, niveles educativos- se hallan insertas en un sistema educativo que está interrelacionado con factores como el control político, nepotismo y demás características cliente- lares de nuestro sistema político. Además, se pierden de vista las interrelaciones con otras secretarías de Estado, con sus partes constitutivas que inconexas simplifican la toma de decisiones del gobierno. En este sentido, lo uno y lo múltiple quedan relegados a una jerarquización asociada a las grandes estrategias de la inteligencia ciega (Morin, 2008), esto es, a los políticos hiperespecializados, que se concentran en obtener indicadores derivados de criterios lineales y con claro enfoque cuantitativo. Es así como sistemáticamente se simplifica la enseñanza a la obtención de indicadores aislados y no necesariamente representativos. No hay que perder de vista que el enfoque complejo, refleja la incompletud en los procesos, pero también permite construir, correlacionar, religar (Morin, 2004).

2. Holograma. Es importante porque va en contra de la idea de solo ver la parte (reduccionismo) o el todo (holismo). El holograma ve el todo y la parte. Esta disyuntiva es clave en la educación en México, pues los tomadores de decisión solo ven lo macro y lo micro de manera aislada, privilegiando lo primero. Un enfoque hologramático permitiría una reciprocidad al interior y exterior de los distintos niveles educativos. Esta es la pertinencia de hablar de política cuando se habla de educación, pues si no hay reciprocidad en la política, tampoco la habrá entre las partes y el todo.

3. Retroactividad. La no causalidad lineal implica que, si bien una causa genera un efecto, 
este retroactúa la información en la causa. En esta tesitura, el efecto ya no es dependiente de la causa sino que abre la posibilidad de autonomía sistémica. Este principio es tanto positivo como negativo; en el primer sentido, implica una ruptura que puede acabar con el sistema (es positivo porque hay ruptura, es decir, posibilidad de origen); en el segundo, reduce la desviación impidiendo el develamiento del origen (por eso es negativo). La simplificación de los indicadores de los modelos cuantitativos son en sí consecuencia de comportamientos diversos, sobre todo político-económicos, pero también son la causa de nuevas reflexiones que permiten ser ubicados como un principio disruptor positivo, capaz de lograr una nueva causa. A nivel de política pública, ello es fundamental, pues la deliberación, genera un incesante flujo de información, que no necesariamente se traduce en una reciprocidad en la toma de decisiones políticas.

4. Recursividad. Es un proceso en el que los efectos o productos son causantes y productos del proceso mismo. Los estados finales generan el propio estado inicial. Asociado al anterior principio, los métodos cuantitativos utilizados en educación generan programas -en tanto productos-, y estos permiten su propia redefinición mediante el rediseño de políticas educativas.

5. Autonomía-dependencia. Solo hay autonomía en la dependencia. Los diferentes niveles de enseñanza dependen del sistema educativo controlado por la Secretaría de Educación
Pública, que si bien son relativamente autónomos comparten una dependencia jerárquica y hasta política; sin embargo, lo que obliga a pensar la complejidad trasciende a nivel de enseñanza, pues, actualmente los indicadores que tratan de medir los "avances" en el aprovechamiento educativo, son criterios reduccionistas que dependen de enfoques dominantes (promedios, eficiencia terminal). Aquí el reto es discutir cómo formular indicadores desde el enfoque de la complejidad.

6. Dialógico. Toma en cuenta lo contrario en un proceso, ${ }_{\mathrm{x}}$ no es solo ${ }_{\mathrm{x}}$ sino su contrario, es decir $_{1 / \mathrm{X}}$. Lo uno es siempre dual. Lo uno es también su antagónico. En este caso la enseñanza viene a ser uno con su negación, y la negación es la dificultad política de generar una reforma del pensamiento capaz de potencializar una forma de ver la enseñanza en relación a otros conocimientos. La enseñanza y esa dificultad son una misma unidad.

7. Reintroducción del cognoscente en todo conocimiento. Observador-observado. El observador es parte de lo que observa, la objetividad no puede ser absoluta. Es claro que los policy makers que manejen los modelos cuantitativos, se asuman desde fuera del sistema que busca intervenir, sin embargo, también son parte de ese sistema educativo que busca transformar de manera minimalista.

Con este recuento breve entre los Siete Principios de Morin (1999) y el tema de la 
educación, hemos tocado tangencialmente otro aspecto importante en la obra de Morin, Ciurana y Motta (2003), esto es, la política, lo político y su relación con la educación.

\section{Complejidad, educación y política}

Según Aristóteles, el hombre es un Animal Político, con lo que dejaba en claro que el humano era el único que podía deliberar asuntos públicos en la polis. Con el paso del tiempo ese espacio de discusión quedó en manos de la religión o, mejor dicho, de la Iglesia. En la actualidad, este campo se concentra en pocas manos, que no solo toman las decisiones educativas sino políticas y sociales; el político profesional es el que determina y monopoliza qué es la vida buena para sus representantes. Las grandes decisiones públicas ya no se discuten en público.

Esta separación entre la política, los políticos y los ciudadanos es cada vez más evidente, pero como lo recuerdan Morin y Delgado (2014), para lograr una reforma de pensamiento es necesario antes una reforma de la enseñanza, por ello cobra especial importancia el relacionar educación con política, puesto que solo se puede lograr una reforma de pensamiento en el terreno del diálogo político. El diseño de políticas públicas educativas no puede estar al margen de la ciudadanía, sino más bien repensarse en aquella.

Para ubicar lo anterior y ampliar la explicación sobre la relación entre complejidad y educación, se propone una explicación más detallada a partir del análisis del concreto anunciado, de modo que desarrollaremos sucintamente las tasas de rendimiento en los niveles educativos.

\section{Análisis de las tasas de rendimiento por} nivel educativo como método de observación

En este apartado se realiza una propuesta con la ayuda de un modelo econométrico para la asignación del gasto público en educación, utilizando las tasas de rendimientos de los distintos niveles educativos en México para el año 2012. En este sentido, la acumulación de capital humano y la inversión en educación, como principal fuente de la misma, son consideradas por los economistas como determinantes básicos de los ingresos individuales y como un importante factor de crecimiento económico. Y desde que, en 1994, México se convirtió en el miembro número 25 de la Organización para la Cooperación y Desarrollo Económicos (OCDE), las políticas públicas en los distintos ámbitos son contrastadas con otras prácticas de sus pares internacionales, lo cual hace que el énfasis se fortalezca comparativamente en lo cuantitativo.

La educación permite a los individuos diferenciarse de los demás, al adquirir conocimientos y habilidades que pueden ser valorados en el mercado laboral. Así, aquellos que posean un mayor y/o mejor nivel educativo, tendrán mayores probabilidades de obtener un salario mejor remunerado, acceso a mejores condiciones laborales y sufrirán menos desempleo (Wooldridge, 2008).

Por su parte, el capital humano puede enten- 
derse como el conjunto de recursos (acceso a los servicios básicos de educación, nutrición, capacitación, experiencia laboral, etcétera) que permiten mejorar la productividad y por tanto los ingresos a nivel individual y agregado. $\mathrm{La}$ acumulación de capital humano se complementa con otras formas de inversión para generar el crecimiento de una economía como condición necesaria para seguir reproduciendo dicho capital (Romer, 1989; Griliches, 1996).

Desde el punto de vista económico la educación es, entonces, una inversión que realizan los individuos con el fin de incrementar su productividad y/o ingresos esperados en un futuro, cuando se incorporen al mercado laboral. En efecto, las investigaciones realizadas en torno al nexo existente entre educación e ingresos, calculan los retornos a la escolaridad por ser esta un indicador de su productividad y un incentivo para que los individuos inviertan en su propio capital; de esta manera, si el incentivo a invertir es bajo, las personas podrían decidir no hacerlo y las brechas salariales entre diferentes tipos de perceptores se podrían incrementar (Psacharopoulus \& Woodhall, 1985).

El planteamiento más utilizado para estudiar los efectos de la inversión en capital humano sobre los ingresos individuales es el de Mincer (1974), que incorpora los supuestos clásicos de la teoría del capital humano y recoge el costo de oportunidad como costo privado de la inversión en educación. Este planteamiento sienta las bases del análisis de los rendimientos de la inversión en educación a partir de una sencilla ecuación donde la variable dependiente es el salario expresado en logaritmo y las variables explicativas son la educación, la experiencia y su cuadrado. Bajo ciertas condiciones, el parámetro ligado a los años de escolarización puede interpretarse como la tasa de rendimiento de un año más de estudio.

Por la sencillez del modelo, al obtener las estimaciones con el método de Mínimos Cuadrados Ordinarios (MCO, en adelante), se presentan diversos problemas en su forma funcional porque la estructura no caracteriza la distribución conjunta del ingreso, edad y escolaridad (Card, 2001). Los tres principales problemas son: 1) el sesgo por habilidad; 2) el sesgo por errores de medición en las muestras si la información no se refiere precisamente a la asistencia educacional; 3) el suponer rendimientos lineales e idénticos a la educación.

Como puede observarse, la linealidad causaefecto entre el ingreso monetario aplicado a la educación, no necesariamente genera los efectos esperados al dejar de lado la complejidad implícita en las habilidades que un alumno puede aprender en el proceso educativo. $\mathrm{Si}$ bien el modelo MCO muestra que ello se debe a la limitante de la distribución conjunta, la medición queda necesariamente incompleta pues presupone que los conocimientos y habilidades adquiridas de un alumno se relacionan necesariamente con la variable ingreso. 
El modelo MCO considera la escolaridad como determinada exógenamente, lo que genera un sesgo por habilidad en las estimaciones y afecta la decisión de invertir más años en educación. Lo que demuestra la evidencia empírica es que el rendimiento escolar es heterogéneo por factores como la diferencia de las habilidades innatas de los individuos, el contexto familiar y la zona geográfica o territorial donde viven (Card, 1998); por lo anterior, posteriores investigaciones incorporan el sesgo por habilidad para tratar de encontrar las diferencias en el ingreso entre distintos individuos mediante variables proxy (Griliches, 1996).

En México existen tres niveles educativos: básico (desde preescolar hasta secundaria), medio (preparatoria) y superior (desde licenciatura hasta posgrado), que están asociados con diferentes niveles de ingresos y rendimientos (Reza \& Manfor, 2000). Pero, si se supone que los rendimientos a la escolaridad son lineales e idénticos para toda la población, no se toma en cuenta que los costos marginales a los que se enfrenta cada individuo por asistir a la escuela no son iguales y su restricción de liquidez impacta en la decisión de invertir más años en educación.

Si bien las políticas educativas deben mostrar su efectividad y eficiencia, los factores lineales de su diseño obstruyen la comprensión compleja del fenómeno. Queda claro que un sujeto -en tanto alumno- carece de características similares con sus pares y, no obstante, queda homogeneizado en generalizaciones que no permiten comprender la diversidad implícita en el proceso educativo. Esto lo podemos ver a la luz del principio de retroactividad, pues al establecer una relación entre causa (ingreso) con un efecto (nivel de aprovechamiento, rendimiento educativo), resulta evidente que el MCO no da cuenta de la retroactividad en las variables, sino que hace a la segunda dependiente de la primera sin posibilidad de una retroactividad positiva.

Como ya se advirtió, el primer modelo desarrollado por Mincer (1974) tiene ciertas limitaciones que hacen que sus resultados sean solo una primera aproximación a la medición del rendimiento educativo. Por tal razón, se han desarrollado metodologías alternativas a la del modelo MCO para encontrar una mejor estimación del rendimiento a la escolaridad. como el Método de Variables Instrumentales (MVI) o el de Máxima Verosimilitud (MV) (Angrist \& Krueger, 1991). La característica de este tipo de estudios es que obtienen rendimientos promedio a un año adicional de escolaridad. Pero si bien esto es importante, no deja de ser un agregado mínimo que no rompe la linealidad del método.

A pesar de estas limitaciones y después de 50 años, dicha forma de estimación ha sido utilizada por varios autores que la han adaptado con base en las características del mercado laboral de los países para los cuales se han llevado a cabo los estudios. Para su análisis, a fin de poder comparar las tasas de rendimiento de la educación, se realizarán algunas adaptaciones de cálculo a la 
ecuación original; es importante señalar que la relación entre educación e ingresos es dinámica, porque el ingreso actual de una persona está explicado por acontecimientos e información del pasado y tiene la capacidad de influir en la dotación de recursos en el presente y futuro.

Esto lleva la discusión a otro plano, pues al utilizar estos métodos (que han venido transformándose, pero solo insertando variables que responden al mismo criterio de acumulación cómo fin) en el campo de lo laboral, vemos cómo se simplifica la educación a un fin laboral, esto es: aquellos que adquieran una educación "correcta" tendrán acceso al puesto laboral "correcto", dicho de otro modo, lo laboral "explica" lo educativo, lo hace dependiente, lo convierte en residual. Tanto trabajo (en actividad no solo remunerada) como educación quedan así simplificados a un asunto de ingreso.

La fuente de información estadística que se utiliza en esta investigación son los micro-datos de la Encuesta Nacional de Ingresos y Gastos de los Hogares (ENIGH) del año 2012, que proporciona el Instituto Nacional de Estadística y Geografía (INEGI). Uno de los principios de la teoría del capital humano propone que, a mayor nivel de educación del individuo, este puede alcanzar mayores niveles de ingreso. En el caso de México, al comparar el promedio de ingresos mensual por edad, nivel de escolaridad y género para el año 2012, encontramos que en las personas que no tienen instrucción el nivel de ingresos promedio más alto fue de $\$ 2,500.00$; los que cuentan con primaria alcanzaron un máximo de $\$ 3,600.00$; los de secundaria, de $\$ 5,900.00$; quienes poseen educación media superior alcanzaron un nivel máximo de \$7,700.00, los de educación superior, $\$ 12,000.00$; y quienes poseen educación de posgrado, $\$ 20,000.00$.

Tabla 1. Rendimientos por nivel educativo

\begin{tabular}{|c|c|}
\hline Nivel educativo & Tasas en \%, 2012 \\
\hline Primaria & 11,2 \\
\hline Secundaria & 17,6 \\
\hline Bachillerato & 14,2 \\
\hline Universidad & 16,2 \\
\hline
\end{tabular}

Fuente: Elaboración propia con el uso de los micro-datos de la ENIGH, 2012

Los resultados de la Tabla 1 indican que el nivel educativo que mayor rendimiento* ofrece a los individuos es el nivel de secundaria, con $17,6 \%$, aunque no tan alejado del nivel universitario, con un $16,2 \%$; los que menor rendimiento ofrecen son el nivel primaria y bachillerato con $11,2 \%$ y $14,2 \%$, respectivamente. El análisis cuantitativo con el modelo econométrico indica que el nivel de educación secundaria es el más rentable y al que se necesitan destinar mayores recursos.

Nuevamente queda de manifiesto cómo el conocimiento se relega a un asunto de rendimientos, perdiendo la riqueza que habría si existiera una reintroducción del cognoscente en el proceso educativo. Para ello, habría que ligar y asociar la causa y efecto, de tal modo

\footnotetext{
* Tomar decisones de política pública, a partir de preguntas como ¿en qué nivel educativo invertir más? permite que el concepto de "rendimiento" se vuelva predominante, pues según los cálculos, se justifica la inversión por nivel educativo.
} 
que el producto sea también productor, para de esa manera vincular lo uno con lo múltiple, y no solamente con el criterio simplificador de la ganancia que representa el capital humano.

\section{Crítica al uso de los métodos cuanti-} tativos en el análisis educativo, así como sus implicaciones de política pública

Como pudimos observar existe en principio una idea causa-efecto entre el capital humano y los rendimientos educativos, esto es, a mayor inversión del primero, mayor incidencia en el segundo, por lo tanto, esta doxa dominante señala que para tener mayores ingresos hay que saber en dónde invertir.

Los resultados de comparar nivel de ingresos promedio (por mes) y nivel escolar afirmaban la correlación anterior, es decir, a mayor nivel de estudios, mayor ingreso. No obstante, la Tabla 1 señala claramente que el nivel secundario es el que ofrece más rendimientos a los individuos. Si bien el lente econométrico que ello establece parte de una racionalidad, no se puede afirmar que la ausencia de otros factores sea determinante para modificar las variables y el modelo mismo. Tal como dice Zemelhann (1997, p.2):

(...) cada vez más los problemas de la sociedad, de la naturaleza, son más difíciles de abordar en los límites estrictos del viejo sistema clasificatorio de la ciencia clásica; cada vez más la realidad rompe con los límites clasificatorios de la disciplina científica.

La economía, por ejemplo, que presume ser una de las ciencias sociales que integra las matemáticas en su análisis, se está enfrentando a problemas cada vez menos económicos, porque se ha distanciado de las condiciones sociales, históricas, culturales políticas, psicológicas y ecológicas que, paradójicamente, son inseparables de las actividades económicas:

La economía, por ejemplo, que es la ciencia social matemáticamente más avanzada, es la ciencia social y humanamente más atrasada puesto que se ha abstraído de las condiciones sociales, históricas, políticas, sicológicas, ecológicas inseparables de las actividades económicas. Por eso sus expertos son cada vez más incapaces de interpretar las causas y consecuencias de las perturbaciones monetarias y bursátiles, de prever y predecir el curso económico incluso a corto plazo. El error económico se convierte, entonces, en la primera consecuencia de la ciencia económica. (Morin, 1999, p.19)

Los policy makers educativos cada vez se enfocan más en el diseño, generación y producción de investigaciones empíricas que buscan resolver casi cualquier problema; no obstante, la evidencia muestra que todavía no han sido capaces de interpretar las causas, consecuencias y efectos que tienen sus políticas públicas. El quid del asunto es que los métodos cuantitativos para el análisis educativo se han enfocado más en el desarrollo de técnicas, que no necesariamente van a la par de un desarrollo teórico y conceptual, de modo que

(...) tenemos una ciencia humana que desde el punto de vista de las estructuras 
conceptuales es relativamente pobre, o que ha permanecido relativamente estática, y que, sin embargo, coexiste con un enorme desarrollo técnico y esto es algo que se tiene que analizar. (Zemelhann, 1997, p.2).

Por lo anterior, el proceso de las políticas públicas educativas queda subordinado a los parámetros y lógicas de los instrumentos técnicos de los métodos cuantitativos.

Pero esto va más allá aún: en la mayor parte de las investigaciones empíricas en el ámbito educativo lo que se busca conocer es la relación construida analíticamente entre educación y pobreza, cuyo vínculo está fundamentado en la causalidad bidireccional observada entre ingresos y educación donde el hogar se toma como unidad de análisis.*

La educación, desde el punto de vista económico, es considerada como una inversión, un "capital humano" que figura entre los activos más importantes que pueden adquirir los hogares. Además, este desarrollo teórico y conceptual está sustentado en los planteamientos de la teoría neoclásica, donde, maximizando una función de utilidad sujeta a alguna restricción -por ejemplo, de ingresos- se analiza la forma en que se interrelacionan matemáticamente las variables involucradas para explicar el fenómeno educativo (Checchi, 2006).

* La razón de utilizar al hogar como unidad de análisis es por las fuentes de información estadística disponibles, por ejemplo, en el caso de México es la Encuesta Nacional de Ingresos y Gastos de los Hogares (ENIGH).
El problema con los modelos neoclásicos es que pueden ser insuficientes para abordar de manera teórica fenómenos multidimensionales como la educación, ya que, aun cuando brindan un marco de referencia importante para conocer ciertos aspectos económicos relacionados con los hogares, se basan en el supuesto del "hombre económico racional", que no resulta del estudio de la conducta humana sino de una construcción matemática unidimensional (Llamas, 2007).

El supuesto de que los agentes buscan maximizar una función de utilidad o bienestar, restringe el estudio del comportamiento de los hogares y las posibles interacciones que se puedan dar dentro del mismo o con sistemas externos a este (el sistema educativo, político, entre otros); en este marco no hay espacio para la elección entre los agentes porque la función de utilidad es una construcción matemática preestablecida. El problema es que los modelos neoclásicos tienen la limitante de que toman el hogar como unidad homogénea, como un todo donde se maximiza una función de utilidad con vectores de consumo, ocio y otros muchos aspectos. Por lo tanto, no se toman en cuenta las posibles interacciones entre los miembros de una familia y se omite una parte importante de la estructura de los hogares: la posibilidad de conflicto, la obtención y división de los recursos, el reparto de actividades, la negociación y la capacidad de aprendizaje y retroalimentación interna y con el entorno (Llamas, 2007).

Esto implica pensar que todos sus miembros se comportan de manera homogénea, que no existe diferencia entre ellos, como por ejemplo, 
de género o edad, y dada una función de utilidad sujeta a ciertas restricciones, elijen una canasta óptima de producción, consumo y ocio. Como puede verse al aplicarse a la realidad económica y social, este tipo de análisis resulta limitado, ya que no se aproxima a lo observado y omite las relaciones e interacciones entre los diversos elementos que son necesarios para comprender los fenómenos educativos.

Ravelo (2012) señala que los rendimientos académicos deben estar más relacionados con factores contextuales y socioafectivos, incluso habla de la inclusión de elementos sobre la historia del sujeto, todos ellos, determinantes cuando se habla de rendimientos académicos.

\section{Reflexiones finales}

La educación es un fenómeno complejo puesto que sus interacciones muestran diversas formas no lineales, y por su carácter multidimensional (social, política, económica, legal) necesita de políticas públicas que tomen en cuenta varios centros explicativos que responden a multiplicidad de factores.

Si bien la mayoría de veces los modelos de evaluación educativa son diseñados para explicar correlaciones entre variables que permitan al tomador de decisiones optimizar su quehacer, ello se vuelve una limitante cuando reduce la complejidad de los múltiples factores asociados al aprendizaje. Esta hiperespecialización no se ha traducido en una mayor convivencia entre los sujetos involucrados, pues las mediciones con énfasis económico-liberal, han reducido la observación a una relación entre ingresos y aprendizaje que no ha mostrado los resultados esperados.

El saber parcelarizado, que establece la relación entre recursos económicos y rendimientos educativos, ha generado una concentración en el tejido complejo de lo educativo (necesariamente multidimensional), proponiendo la creencia de que esa reducción es racional y que solo habría que tecnificar la forma de observarlo, medirlo, entenderlo y clasificarlo para, con ello, establecer qué ciudadanos han de ser incluidos y cuáles no (también, dentro de un modelo de desarrollo simplificador).

En consecuencia, el reto en materia de diseño de políticas públicas educativas es generar un diálogo fructífero entre los diferentes saberes involucrados en la lógica educativa. Ante ello, cabe preguntarse: ¿es plausible generar indicadores desde el horizonte de lo complexus dinámico?, ¿cómo sería posible?, ¿son los métodos mixtos una alternativa real? Los modelos cuantitativos -en tanto unidad¿tienen la posibilidad de transformar el Todo solamente por el puente de la política? Tal vez antes de plantear respuestas podríamos discutir las posibles preguntas.

Es menester que los hacedores de políticas (en tanto discusión de asuntos públicos entre los agentes involucrados en la educación, política, academia, sociedad, gobierno) recuperen el carácter público de las políticas no solo educativas, sino de toda la política. 


\section{Referencias}

Angrist, J. \& Krueger, A. (1991). Does compulsory school attendance affect schooling and earnings? The Quarterly Journal of Economics, 106(4), 979-1014.

Card, D. (1998). The Causal Effect of Education Earnings. Center for Labor Economics, 3, 1802-1863.

Card, D. (2001). Estimating the return to schooling: progress on some persistent econometric problems. Econometrica, 69 (5), 1127-1160.

Checchi, D. (2006). The economics of education: Human Capital, family background and inequality. Londres: Cambridge University Press.

Griliches, Z. (1996). Education, Human Capital and Growth: A Personal Perspective. Working Paper No. 5426. National Bureau of Economic Research.

INEGI, (2013). Encuesta Nacional de Ingresos y Gastos de los Hogares 2012. Conociendo la Base de Datos.

Llamas, I. (2007). Educación y Desarrollo. En J. L. Calva (Coord.), Educación, ciencia, tecnología y competitividad (pp.19-32). México, D.F: Miguel Ángel Porrúa.

Morin, É. (1999). Los siete saberes necesarios para la educación del futuro. París: Santillana-UNESCO.

Morin, É. (2004). El método 4. Las ideas, su hábitat, su vida, sus costumbres, su organización. Madrid: Cátedra.
Morin, É. (2008). Introducción al pensamiento complejo. Barcelona: Gedisa.

Morin, É. \& Delgado, C. (2014). Reinventar la educación. México: Multiversidad Mundo Real.

Morin, É., Ciurana, E. R. \& Motta, R. D. (2003). Educar en la era planetaria. Barcelona: Gedisa.

Mincer, J. (1974). Schooling, Experience, and Earnings. Human Behavior \& Social Institutions No. 2. New York, NY: National Bureau of Economic Research.

Psacharopoulus, G., \& Woodhall, M. (1985). Education for Development. An Analysis of Investment Choices. 1era. edición. Londres: Oxford University Press.

Ravelo, E. (2012). Calidad, aprendizaje y rendimiento académico en educación superior. Revista Educación y Humanismo, 14(23), 17-36.

Reza, A. y Manfor, L. (2000). Non-Linearities in returns to education in Lybia. Education Economics, 9 (2), 139-144.

Romer, P. (1989). Human Capital and Growth: Theory and Evidence, NBER. Working Paper No. 3173. Cambridge M.A.: National Bureau of Economics Research.

Wooldridge, J. M. (2008). Instrumental variables estimation of the average treatment effect in the correlated random coefficient model. In T. Carter, L. Millimet, J. Smith, E.

Zemelhann, H. (1997). Epistemología y criterios de calidad en investigación. Conferencia Magistral impartida en la Facultad de Estudios Profesionales, Iztacala, UNAM. 